\title{
TULSI: an NLP System for Extracting Legal Modificatory Provisions
}

\author{
Leonardo Lesmo ${ }^{\circ}$, Alessandro Mazzei $^{\circ}$, \\ Monica Palmirani ${ }^{\star}$, Daniele P. Radicioni ${ }^{\circ}$ \\ ${ }^{\circ}$ Dipartimento di Informatica, Università di Torino \\ ${ }^{\star}$ CIRSFID, Università di Bologna \\ \{lesmo, mazzei, radicion\}edi.unito.it \\ monica.palmirani@unibo.it
}

\begin{abstract}
In this work we present the TULSI system (so named after Turin University Legal Semantic Interpreter), a system to produce automatic annotations of normative documents through the extraction of modificatory provisions. TULSI relies on a deep syntactic analysis and a shallow semantic interpreter that are illustrated in detail. We report the results of an experimental evaluation of the system and discuss them, also suggesting future directions for further improvement.
\end{abstract}

\section{Introduction}

Intelligent indexing, querying, searching, filtering, retrieving and annotating the ever increasing amount of legal text documents is a major challenge in the field of Artificial Intelligence and Law concerned with Natural Language Processing. In the past decade many efforts have been invested in Information Extraction, that investigates the automatic extraction of salient information from legal texts (e.g. normative references, metadata, etc.), to devise tools and techniques to face this challenge [5, 26, 16, 40]. Some information extraction systems analyze only the surface of the texts, e.g. individuating 'trigger' words, and then attempt to fill out a fairly simple form that represents the objects or events of interest. An example task is that of electronic feeds, that are examined to see if they contain some target terms, and therefore deserve further analysis [21]. However, there exist many tasks that require more sophisticated approaches such as semantic entailment [18], and textual inference [20]. The aspects peculiar to the legal domain make it difficult to distinguish commonsense speech from the juridical lexicon, and the NLP techniques have to be applied with some more warnings and cautions [35]. This paper illustrates how it is possible to take into consideration the above mentioned peculiarities of the legal text and to foster the NLP techniques and tools, especially using the regularities of legal language.

One major obstacle to the automatic managing of documents is the "natural language barrier", i.e. the translation of a sentence into some form of semantic repre- 
sentation [25]. This difficulty becomes a real barrier in the legal text, due to several peculiarities of the legal language. The large use of rethoric in the legal domain (e.g. metaphor and similarity), the presence of technical syntagms in the lexicon with meanings different from common sense [34], the integration with foreign terminologies and concepts ${ }^{2}$ the peculiar cultural and legal tradition of each legal system (e.g. civil law vs. common law), the implicit norms and the legal drafting techniques with their side effects (e.g. undefined reference), the ambiguous and vague legal language that is sometimes a drafting technique for guaranteeing the long-term persistence and flexibility of some general and abstract fundamental rights [33]. Natural Language Processing (NLP) approaches language complexity by considering several layers of analysis. The most critical and complex layers are concerned with the syntactic and semantic analyses of a sentence. How accurate are such analyses required to be? From a theoretical perspective only "deep" analyses are able to capture the full meaning conveyed by a sentence. From a practical perspective, the answer depends on the specific problem that we need to solve, and "shallow" approaches can be well-suited.

Information extraction techniques are often used to extract information that is useful to semantically annotate texts. This is the focus of the present work that addresses the problem of semantic annotation of legal texts. In this domain, systems automatically identify and classify structural portions of legal documents and their intra- and inter-references [10, 29], and that produce semantic analyses [36, 38]. Various initiatives have been established at the national and international levels to devise XML standards that describe legal sources, and to devise schemas that allow encoding such legal documents [24]. Although highly valuable, a human annotation process is expensive and error-prone 3 Several tools were developed in the last decade for minimizing manual annotations using parsers to detect normative references and legal text structure [32, 9, 17]. Such efforts will fully be exploited in conjunction with NLP tools to extract structural and semantic data from legal texts.

\section{Organization of the Work}

The work focuses on a subset of all possible semantic meta-data annotations of the text, and namely the annotation of modificatory provisions. A modificatory provision is a fragment of text that makes a change to one or more sentences in the text or in the normative arguments (e.g. time of efficacy of the norms) or in the range of application of the norm (e.g. extension, derogation, interpretation). Furthermore, a modificatory provision can affect the entire text along with its annexes, e.g., as in case of repeal of an entire law. Finally, a modificatory provision can affect legal relations that hold among the provisions of a legal system, so the text is not so much involved as an effect of the

\footnotetext{
${ }^{1}$ E.g., the technical syntagm "digital signature" is at the same time a legal concept and also a definition of a particular technology.

${ }^{2}$ E.g., the term "privacy" is a well-known legal term that represents a specific legal concept in all EU jurisdiction; in US there is a different legal meaning, in Italy this term is not used in any legal document about this topic.

${ }^{3}$ In particular, it is sometimes necessary to apply modern legal interpretation principles that go beyond the textualism theory, and that integrate the objective literal interpretation with teleological scope, socioeconomical goals and historical-cultural elements.
} 
modification. This is the case in which a decree-law is converted into law: it is possible that the text is not changed, but the legal validity of the text assumes a permanent legal status. Other relevant modificatory provisions affect the temporal argument of the norms: it is possible to make it retroactive the effect of the norm. Modificatory provisions are particularly relevant, since they affect the whole normative system 4 It should be considered, in this regard, that a lavish use of normative modifications tends to undermine the certainty of law, so that the changes are sometimes fragmentary and incoherent, making it even more difficult to clearly understand what is the law, or which provision version counts as law. Any modification made to a single proposition can in principle affect other propositions as well; as a consequence, handling modificatory provisions is a rather thorny task. Automating the process of semantically annotating modificatory provisions -or even assisting human annotators in their work- would be of great help in simplifying the normative system and in consolidating texts of law. Consolidated text is the updated version of a normative text, embodying the changes.

In this paper we describe TULSI, an NLP system that combines deep syntactic analysis and shallow semantic interpretation of natural language, in order to enhance the NIR (NormaInRete Italian Government XML standard) annotation with semantic metadata. The system uses the Turin University Parser (TUP, [23, 22]) to build deep syntactic structures of sentences, and a rule-based semantic interpreter to fill the frames representing the shallow semantic content of sentences containing modificatory provisions. From a theoretical point of view, this research explores whether a combination of deep syntax and shallow semantics is well-suited in some specialized domains, such as the legal texts containing modificatory provisions, in which the language is more regular and controlled. This allows the use of a specialized legal and linguistic background knowledge, that has been formalized as an ad-hoc taxonomy of the modificatory provisions [31]. From a practical perspective, this research provides human and automatic annotators with a tool that can greatly speed-up the annotation of semantic meta-data in normative documents. The TULSI system can be plugged into the Norma-Editor [28], a software module (developed by CIRSFID-University of Bologna) used by the High Court of Cassation of Italy since 2005 for marking up all the legal documents of the Italian Official Gazette (now including about 70,000 documents). Currently semantic annotations of modificatory provisions are made manually by a legal expert of the Court, and the TULSI system can be useful to reduce and/or point out human annotation errors, to harmonize the different mark-up styles, and to reduce time required by routine annotations.

This article is structured as follows. We first survey some closely related work, and point out similarities and differences to our approach (Section 3). We then introduce the representation format of modificatory provisions: the NIR XML representation is briefly illustrated, and the taxonomy of modificatory provisions is presented (Section 4). We then introduce the TULSI system: the preprocessing step (Section 5.1), the parsing step (Section 5.2) and the semantic interpretation step (Section 5.3). Next, we report an experimental assessment of the system done on the High Court of Cassation XML documents: we describe the experimental setup and the evaluation metrics

\footnotetext{
4"We can now define the concept of a normative system as the set of all the propositions that are consequences of the explicitly commanded propositions." [3]
} 
adopted (Section 6.1), and we report the results and discuss them in detail (Section 6.2). In Section 7] we draw some conclusions and outline possible directions for future work.

\section{Related Work}

The work described by McCarty [25] uses a deep syntactic parser (namely, Collins' parser, [15]). This parser is used to build a full syntactic description of legal sentences, and produces constituent structure. Moreover, McCarty uses a logic language, i.e. a deep semantic structure, to represent the entire semantics of sentences, rather than focusing on semantic frames.

To the best of our knowledge, the very first attempt to extract textual modificatory provisions from legal texts is the work described in [6, 7]. The system Themis was designed to simplify and speed up the legal drafting process, with particular emphasis on rewriting (or rebuilding) textual amendments. The system is concerned with the automatic generation of amending texts embodying the changes described by the legislative drafter. In particular, it allows drafters to encode textual modificatory provisions in a so-called Change Description Document and then to generate an amending act which reflects those changes. Arnold-Moore uses a frame-like representation to encode the meaning of modificatory provisions, and an SGML representation that shares some aspects with our input encoding, but on the whole, this system can be hardly compared with TULSI. Arnold-Moore's approach is called "indirect consolidation" [27] that in some legal traditions, like the Italian one, is not legally binding. For this reason it is not an interesting technical comparison with our work.

The work by Bolioli and colleagues [11] focuses on automatically recognizing and encoding modificatory provisions. It has been a pioneering one, in which the authors investigated at the same time how to extract intra-document citations and how to convert the norms from a textual format into an XML format, given a specific DTD. A pilot-case is considered of about 100 modificatory provisions from a corpus of 8 Italian laws 5

A project that has some commonalities with our research is SALEM [8, 9, 38, 14, 39]. SALEM automatically annotates the modificatory provisions of NIR documents by using syntactic parsing and uses a rule-based strategy to fill the semantic frames. SALEM is based on a shallow syntactic analysis is produced by a chunk parser. By using a deep parser, we can cover the analyses of a wider range of syntactic phenomena that cannot be accounted for in a pure chunk approach, as coordination or relative clauses. Similar to our approach, SALEM uses a provision taxonomy to build the semantic frame. However, SALEM produces classification and analysis of general provisions, whilst our project is concerned on more specific provisions, that is modificatory provisions. As a consequence, our taxonomy is specifically drafted for modificatory provisions. In all the mentioned works, SALEM has been subject to an experimental evaluation similar to ours. However, it can be hardly compared to ours for two reasons: the dataset used in the experimentation is not publicly available; secondly, the measure

\footnotetext{
${ }^{5}$ Also, since the Italian standard NormeInRete was being devised, the paper [11] provides the first description of a software system for the automated mark-up of Italian legal texts, funded by the AIPA (the Italian Authority for promoting the information technologies in the Italian Public Administration).
} 
therein employed considers the number of XML tags correctly identified, whilst we consider the number of modificatory provisions correctly identified (see Section 6 .

A recent work on the topic of automatic consolidation has been proposed in [26]. This work proposes a pattern-matching based approach analysing text that uses a basic XML preprocessing, but without using a parser. The underlying hypothesis about this approach is that Japanese syntax for amendments is very regular and considered unambiguous by experts. As a consequence, they empirically show that sixteen regular expressions are sufficient to capture and consolidate modificatory provisions, since the accuracy of this system closely approaches $99 \%$ for both precision and recall. We hypothesize that such results can be explained from the regularity of Japanese, which is a case-based language. Also a simpler structure of XML documents can explain such accuracy.

\section{Domain Representation}

The set of considered documents was previously marked up using NormeInRete (NIR) XML standard for Italian legislative documents [28] by legal experts (ten operators, three supervisors) of the High Court of Cassation between 2005-2009, using NormaEditor. Norma-Editor is a specialized word processor for legal documents; it was used to automatically detect the structure of the act and the normative references, according with the NIR main principles design, using an interactive human computer interface module that permits a gradual refinement by the operator during each step of the document analysis. For this reason after the automatic detection done by a set of parsers of Norma-Editor, in case of errors, the end-user refines the annotation manually. Norma-Editor detects the normative references with high precision (about $99 \%$ accuracy), based on the interviews to operators and considering the complete citations [32]. After this step, legal experts manually qualify the citations involved in the modificatory actions (insertion, repeal, substitution, suspension, derogation, etc.) in order to complete the markup of the fundamental anatomy parts of a modifications (see Section 4.1).

In particular, $70 \%$ of the overall time dedicated to markup of an act is invested in verifying normative references (correctness and completeness), to interpret the legal text for deciding the type of modification, and to qualify them using the NIR taxonomy of modifications (see Section 4.2). Due to the amount of time dedicated to this phase of the work, the supervisors of this specific task (three people in High Court of Cassation) declared, in a feedback interview, that the quality of the markup done by their operators, in the end, is really good and it usually does not need further refinements. The major problems were identified in the temporal modifications where the linguistic elements of the sentence need a strong legal interpretation, and also the temporal parameters (open intervals, undefined period of time, duration conditionals) are not easily detectable by neither automatic nor manual procedures.

After this supervised markup, Norma-Editor converts the internal format into the NIR XML standard syntax. NIR encoding provides a definition for the main legal parts of a legislative document:

${ }^{6}$ The files containing the DTD are available at the URL: http://www.digitpa.gov.it/ standard-normeinrete 
1. main structure elements (preface, preamble, body, conclusions) including very granular sub-provisions like paragraphs, subparagraphs, letters and numbered items; an example is

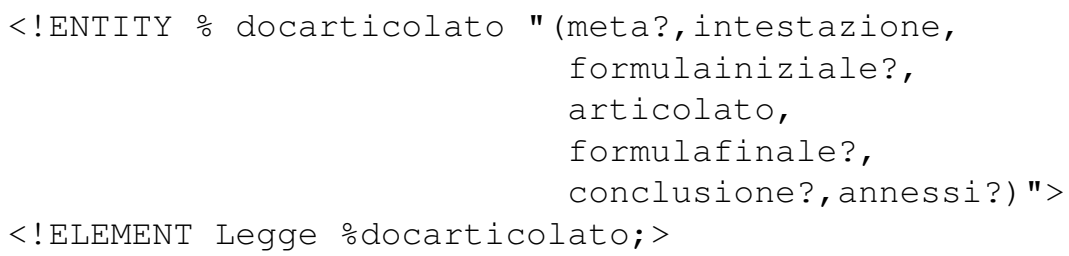

2. inline qualified part of the text (e.g. normative references, definitions, etc.); an example is

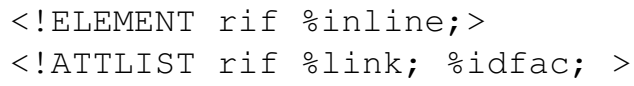

3. legal metadata for annotating the interpretation made by the legal expert during the markup (e.g. modification metadata).

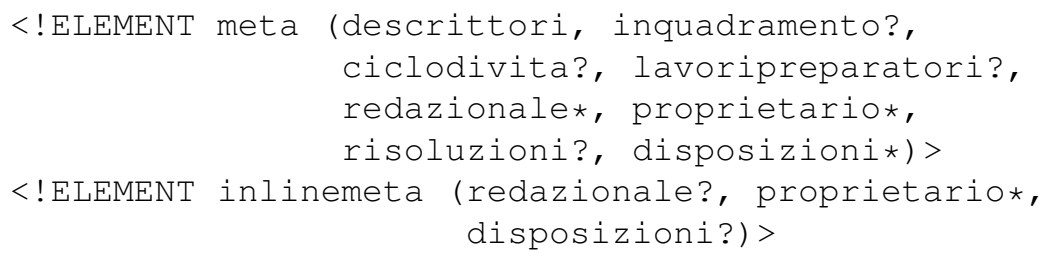

\subsection{Anatomy of Modificatory Provisions (XML NIR)}

A modificatory provision can be qualified through a specially defined space called $\langle$ meta, in which a Uniform Resource Name (URN) connects the element expressing a qualification with the textual element referred to (be this an atomic element, or a text string). A modificatory provision includes the following elements [30]:

- ActiveNorm: the provision that states the normative modification;

- PassiveNorm: the provision that is affected by the modification;

- Action: the action produced by the active provision on the passive norm;

- Times: the time of application of the modificatory provision;

- Content: the element that models the old text to replace or repeal in the modified provision, as well as the new text inserted in the destination. Recently the NIR standard models also the position in the text where the modification has to be applied -e.g., after the words "Ministry of Justice" include "and Ministry of Interior"- with the tag dsp:posizione ("posizione' is the Italian word for 'position'); 
a)

All'articolo 40, comma 1, della legge 28 dicembre 2005 , n. 262, le parole: "sei mesi" sono sostituite dalle seguenti: "dodici mesi". At article 40, comma 1, [...] the words: "six months" are substituted by the following: "twelve months". b)

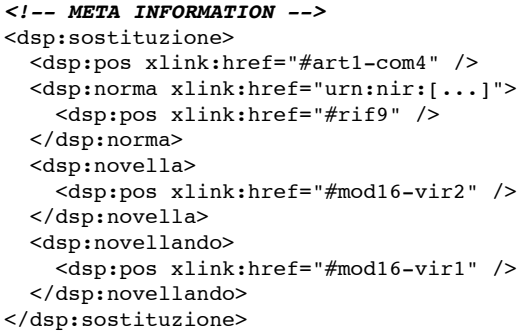

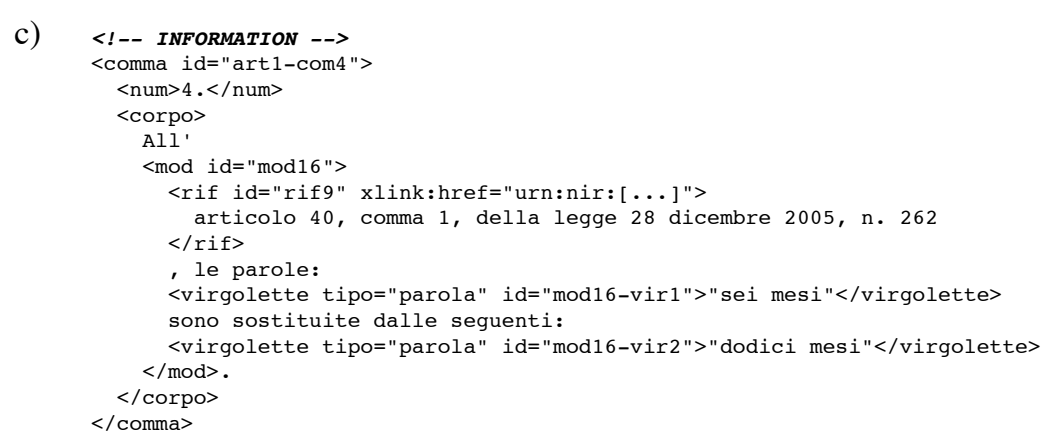

Figure 1: Example of encoding for a substitution provision: in $a$ ) the input sentence is displayed; in $b$ ) we report the metadata describing the salient elements of the modification; in $c$ ) we illustrate how the modification is actually annotated in the XML-encoded text.

- Purview: a part used to describe the scope of application of the modification, as by specifying any exception, extensions, or authorized interpretations (e.g. derogation for the earthquake victims, extension for the teleworking employees);

- Space: a function used to specify a geographical area to which the modification applies (e.g. applicable only to the Sicily Region);

- Conditions: where a modification is an effect dependent on a conditional (e.g. event like a war or a class of circumstances).

The NIR standard includes in its Document Type Definitions a specific part dedicated to modifications [2]. Figure 1 illustrates how a non-qualified provision can be enriched with semantic metadata. Semantic metadata are linked to structural elements by a URN to assert the kind of modification (action), the active and passive norms, and other subelements describing the action. Several classes are used to qualify the behavior of modificatory provisions: these classes are identified by the namespace $d s p$, so named after the word 'disposizioni' (provisions). Every class of modificatory provisions is modeled through a number of sub-elements that further specify it. In the example in Figure 1, the tag dsp:substitution, linked to the text of the modificatory provision 
specifies the Action; the tag dsp:norma, linked to the structural element rif (normative reference) specifies the Passive Norm; the tag novella, linked to the structural element virgolette (quoted text) specifies that the Quoted Text should be added in the Active Norm; the tag novellando, linked to structural element virgolette specifies that the quoted text should be overwritten in the Active Norm 7

\section{Encoding of Modificatory Provisions}

Furthermore, thanks to the regularity of the language used in active modificatory provisions, we have individuated a number of very frequent elements. Such elements are often paired to text excerpts that are relevant to fully qualify the modificatory provisions: Date, Quoted text, Position, Condition.

Date. A date can express either the time when a modification is applied (e.g., a modification can be effective immediately or at some later time), or it can modify a term, or signal the beginning/end of a temporal modification (duration of the action, like the suspension).

Quoted text. Text enclosed within quotation marks can be used to define a concept (e.g. definition provision or constitutive norm). Moreover, it can be used in a modificatory clause as text to be inserted into the passive or target document to be modified (the intervening string is called novella). Finally, it can be used to individuate the text being replaced or deleted: in this case, the string is said to be the novellando.

Position. The position is expressed by a couple of elements: function words such as 'before', 'after', 'between', 'from', and 'to', followed by a quoted string or atomic document partition (paragraph, line, index, title). The position denotes the point where a modification occurs in the passive or target text.

Condition. The condition expresses the constraints to the modification: conditions are usually related to an event, to a legal form, or to a place.

Normative texts are described not only through structural elements, but also through metadata, that is additional information provided by editors and publishers to make the content more suitable for interpretation and use. For example, metadata may include the following:

- data for identifying and managing the legislative process traceability (such as publication, location or legislative workflow, etc.);

- temporal attributes (helpful in versioning a norm as it changes over time or for the application of the norm);

- functional metadata (for modificatory provisions and functional provisions, as well as for permissions, obligations, rights, etc.), and content topics.

Metadata can be thought of as a layer at the top of the structural data, not mixed with to the content: they are rather external, and defined through domain ontologies. Enriching a text with labels expressing its semantic content requires a semantic annotation process. Since such process involves many different competences and sources of knowledge, it has been traditionally a complex task, and constitutes a highly difficult task for automatic approaches.

\footnotetext{
${ }^{7}$ Further details about the input format are provided in Section 5.1
} 


\subsection{A Taxonomy of Modifications}

The detailed taxonomy taken as reference for the NLP analysis is presented with full details in [13, 31]. We report here the definitions for the basic categories:

- Changes made to the provision text or form (an integration, replacement, deletion, relocation) or to the provision meaning (an interpretation or variation of meaning);

- Changes made to the range of a provision (an extension of its subject matter or range of application or a provision stating a derogation to the same);

- Changes made to the temporal parameters of the norm (the time of its entry into force, and the time of efficacy);

- Changes made to the legal status of the norm within the legal system (a decreelaw that is converted into law, an international treaty that is ratified into a national legal system);

- Changes made to the powers conferred under a norm within the legal system (examples being a legge delega, used by Parliaments to empower the government by issuing a legislative decree under which certain public laws may be passed; or a legislative decree endorsing a ministry to deregulate a certain subject matter within its competence; or again an EU directive transposed into domestic law).

\section{Annotating Modificatory Provisions}

To annotate modifications with meta-information we have devised a three-step process. In the first step we retrieve the possible location of a modificatory provision within the document, and we simplify the input sentences, so to maintain only text portions that convey relevant information. In the second step we perform the syntactic analysis of the retrieved sentences; in the third step we semantically annotate the retrieved provision by using its syntactic structure and the modificatory provisions taxonomy.

\subsection{Retrieving the Modificatory Provisions}

Although legal documents can be very large, often only small fragments contain information about modificatory provisions. As a consequence, correctly individuating such information is a relevant step, that deeply affects the performance of the whole information extraction system [5]. Consequently, XML documents that are compliant with the NIR DTD, are preprocessed in order to prune irrelevant information. XML structure can be used to prune irrelevant information. Let us consider the modificatory provision contained in the sentence:

All'articolo 40, comma 1, della legge 28 dicembre 2005, n. 262, le parole "sei mesi" sono sostituite dalle seguenti "dodici mesi".

At the article 40, comma 1 of law December $28^{\text {th }}, 2005$ number 262, the words "six months" are substituted with the following "twelve months". 


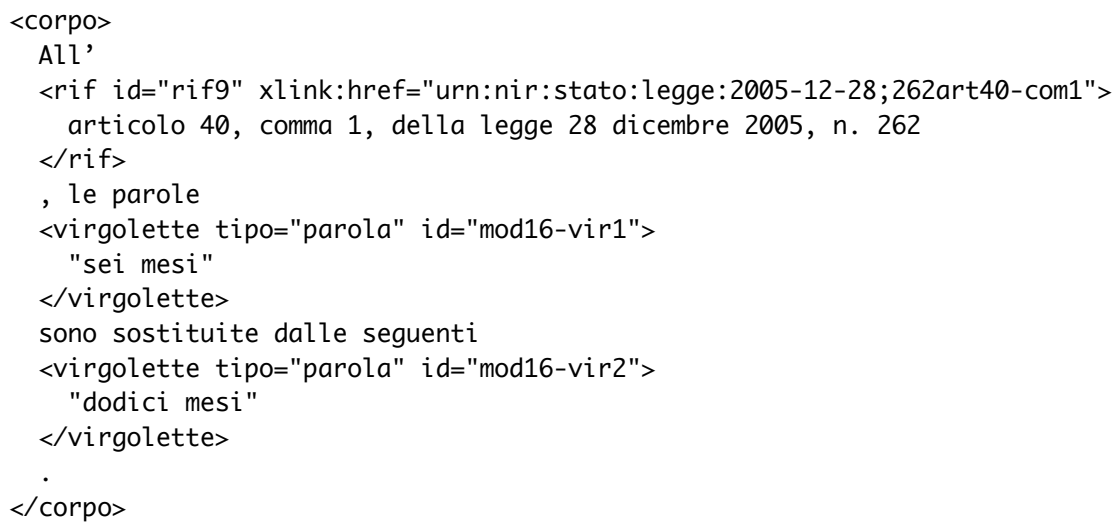

Figure 2: Fragment of a XML NIR document tagged as a $\langle$ corpo $\rangle$, which has as subelements the tags $\langle$ rif $\rangle$, to refer to other sources, and $\langle$ virgolette $\rangle$, to quote excerpts of text.

The XML format corresponding to the input sentence is provided in Figure 2

In accordance with Italian drafting principles [2], we assume that all possible modificatory provisions are included in the body of a norm, which is enclosed between tags $\langle$ corpo $\rangle{ }^{8}$ so we retain the text that is present in such XML nodes, and disregard the remaining elements. An exception to this assumption is the $\langle$ alinea $\rangle$ tag, that is illustrated in Section 5.2.1.

In order to simplify the work of the syntactic parser, we perform two additional rewritings on the text of the body, based on the NIR XML annotation of the document. Namely, we search for the XML elements $\langle\text { rif }\rangle^{9}$ that enclose a text fragment denoting a reference to a particular fragment of a legal document, and we replace the text contained in such XML node with the corresponding identifier. Such constants are hereafter referred to as RIF. Similarly, we search for the XML elements $\langle$ virgolette $\sqrt{10}$ that enclose a quoted text fragment, and we replace the contained text with the corresponding identifier. Such constants are hereafter referred to as VIR. Both rewritings greatly simplify the task of the parser, with no loss of information. By applying the pre-processing step to the XML excerpt presented in Figure 2, we obtain the sentence

All' RIF9, le parole VIR1 sono sostituite dalle seguenti VIR2.

At RIF9, the words VIR1 are substituted with the following VIR2.

\section{Handling frequent annotation errors}

Furthermore we automatically rewrite some idiomatic expressions. Indeed, these idioms can hide the information necessary for the TULSI system to correctly qualify

\footnotetext{
${ }^{8}$ Corpo is the Italian word for body.

${ }^{9}$ Rif stands for riferimento, the Italian word for reference.

${ }^{10} \mathrm{Vir}$ stands for virgolette, the Italian word for quotes.
} 
modificatory provisions. For example, a frequent annotation issue is the following one. Given the input sentence:

e dopo le parole: "interessi passivi dedotti" sono inserite le seguenti: "o non dedotti";

and after the words: "red-ink interests deducted" are inserted the following: "or not deducted", 11

the text is rewritten into:

e dopo le RIF428: VIR1 sono inserite le seguenti: VIR2;

and after the RIF428: VIR1 are inserted the following: VIR2;

The constant RIF428 is used to annotate the word 'words'. Strictly speaking it is used improperly, since the word does not per se constitute a reference. This annotation results in an implicit normative reference [37], which creates a semantic confusion. Such linguistic construction is rather common in the annotated files, due to the fact that there is not other semantic tag or attribute to distinguish the explicit normative reference from the implicit one, so we simplify the sentence through regular expressions. Regular expressions (see also 5.2.1) allow one to describe, find and replace (complex) strings. In particular, the goal of the replacement is to make explicit the reference, and -in the case at hand- to provide it with the correct status of the expression identifying the involved norm. The considered example is thus rewritten into the following (additions in bold):

\section{[...] e al RIF428, dopo le seguenti parole VIRI sono inserite le seguenti: VIR2. \\ [...] and at RIF428, after the words VIR1 the following words VIR2 are inserted.}

Another common problem due to the annotation practice is that sometimes prepositions (such as 'before', 'after', 'at', 'at the words', etc.) or whole idioms are wrongly tagged together with the contiguous reference. In such a case, for example, the sentence 12

Dopo l'allegato 4 è aggiunto il seguente allegato 5: Allegato 5 [. . .].

After the attachment 4 the following attachment 5 is added: 'Attachment 5'[...].

would be rewritten as (salient words formatted in bold):

RIF246 è aggiunto il seguente allegato 5: VIR1 [...].

RIF246 the following attachment 5 is added: VIR1 [...].

Here two different problems typically arise: first, the locative preposition -i.e., a fundamental piece of information - 'after' is lost, hidden in the reference. Accordingly,

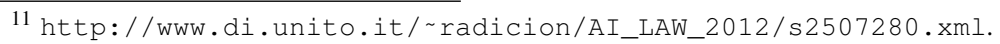
$\mathrm{xml}$

12 Excerpt from the file http://www.di.unito.it/ radicion/AI_LAW_2012/s2603265.
} 
the parser will produce a wrong analysis, confusing the RIF246 with the subject of the sentence, which undermines the whole interpretation process (more about the parsing step in next Section, and about the use of parsing information in Section 5.3. Clearly, this style of annotation prevents the system from correctly extracting the modifications; to overcome such problems we search the beginning of the original string replaced by the RIF tag for a list of prepositions, and if any such preposition is found, it is moved before the constant. In the considered example, the sentence obtained at the end of the preprocessing would become:

Dopo il RIF246 è aggiunto il seguente allegato 5: VIR1 [...].

After RIF246 the following attachment 5 is added: VIR1 [...].

After rewriting the sentences contained in the corpo elements, and after having checked some frequent annotation errors by applying regular expressions, the sentences are parsed by the TUP module.

\subsection{Syntactic Analysis}

TUP is a rule-based parser whose result is the syntactic structure of sentences in the dependency format [22]. Dependency formalisms represent syntactic relations by connecting a dominant word, the head (e.g., the verb 'sleeps' in the sentence Joe sleeps) and a dominated word, the dependent (e.g., the noun 'Joe' in the same sentence). The connection between these two words is usually represented by using labeled directed edges (e.g., subject): the collection of all dependency relations of a sentence forms a tree, rooted in the main verb. A dependent is called an argument of the head if it is mandatory for the correctness of the sentence, or alternatively is called modifier if it is not mandatory. Dependency formalisms are contrasted with constituency-based ones, where syntactic structures are represented by grouping words. Dependencies present some advantages especially for languages where the order of words is relatively free [23].

After morphological analysis and part of speech tagging [22], TUP processes the sentence in three phases: chunking, coordination and verbal subcategorization.

\section{Chunking}

The chunk $\mathrm{s}^{13}$ produced by TUP are complex (in the sense that nested chunks are allowed) and are built by a procedure that applies chunking rules to larger and larger chunks, using a predefined sequence of chunking levels. For instance, adverbial chunks (e.g. [very often $]_{A D V}$ ) are built before adjectival chunks (e.g. [[very often $]_{A D V}$ useful $_{A D J}$ ), which, in turn, are built before nominal chunks (e.g. [[[very often $]_{A D V}$ useful $_{A D J}$ solutions $]_{N O U N}$ ), and so on. Currently, the parser includes 326 chunking rules, that are grouped according to the syntactic category of the head.

\footnotetext{
${ }^{13}$ Chunks can be defined as groups of syntactically related and adjacent words [1].
} 


\section{Coordination}

Coordination notoriously is one of the hardest problems in NL. And coordination abounds in Italian legal texts. We report an example below 14 .

È approvata la proposta formulata dalla Regione Campania, in merito alle domande presentate per il bando del $2003 \boldsymbol{e}$ (coord) riferite alla predetta Regione per le attività estrattive, (coord) manifatturiere, (coord) di servizi, (coord) delle costruzioni e (coord) di produzione e (coord) distribuzione di energia elettrica, (coord) di vapore e (coord) acqua calda.

It is approved the proposal made by the Regione Campania, concerning the applications presented for the advertisement of 2003 and $_{1}$ (coord) referred to the aforementioned Region for the activities extractive, (coord) manufacturing, (coord) of services, (coord) of construction and and $_{2}$ (coord) of production and and $_{3}$ (coord) distribution of electricity, (coord) of steam and $_{4}$ (coord) hot water.

As it can be seen, some coordination is expressed by commas and can be nested. In order to cope with these complexities, coordination is handled by means of a set of heuristics that, when a conjunction is encountered, perform the following steps:

1 Look for the best second conjunct. In our example (in case of composed conjuncts, out of the brackets, the head word): and $\rightarrow$ referred; and $_{2} \rightarrow$ of [production]; and $_{3} \rightarrow$ distribution; and an $_{4} \rightarrow$ [hot] water;

2 Look for possible first conjuncts (many of them; see below for the final choice);

3 Choose the best pair. In our example: and $\rightarrow$ presented; and an $_{2} \rightarrow$ of [construction]; and $_{3} \rightarrow$ production; and $_{4} \rightarrow$ steam.

4 Move back to ascertain if any comma can act as a previous conjunction of a sequence. This enables the system to recognized as conjunctions all commas except the last one (the one before "of steam"). This is due to the presence of the preposition ("of" steam). The system assumes in fact that the two conjuncts are "steam" and "hot water". So, "of electricity" is inhomogeneous with respect to them, and it is not accepted as a previous conjunct.

\section{Verbal Subcategorization}

After the previous steps, the partially built parsing structure includes a set of (possibly very large) chunks, including prepositional modifiers and conjunctions, and some verbs ${ }^{15}$. The various chunks are verbal dependents: they are now attached to the verbs (via rules that take into account distance from chunks and verbs, intervening subordinating conjunctions, relative pronouns, and so on). After this, each verb is associated

\footnotetext{
14 The English pseudo-translation aims to keep the ordering of the Italian words. Conjunctions are labelled with subscripts for reference purposes in the following description.

${ }^{15}$ Actually, some inputs can be without verbs. In this case, either the analysis now includes a single chunk (and the task is completed) or one of the chunks is chosen as the head and the others are attached to it. This is still accomplished via heuristics.
} 
with a set of dependents. The final task, in order to provide the semantic interpreter with all required information, is to determine the labels of the arcs.

This is made by exploiting knowledge about the subcategorization frames of verbs. Each verb is assigned to one or more verbal subcategories; each of them, in turn is associated with a verbal frame. The final goal of this step is to find, for each verb in the sentence, the best match between its dependents (found in the previous steps) and the verbal frames associated with its possible subcategories. The task is made more complex by the existence of transformations, that affect the possible surface realizations of a verbal frame. For instance, the verb "autorizzare" (authorize) has just one possible subcategory, i.e. trans- $a$ (transitive verbs admitting a theme governed -in Italian- by the "a" preposition). Although the base description of trans- $a$ involves a subject, and object and a theme, as in

([Il terzo comma $]_{s u b j}$ autorizza [il Consiglio $]_{o b j}$ [a inviare aiuti alimentari theme $_{\text {) }}$

([The third paragraph $]_{s u b j}$ authorizes [the Council $]_{o b j}[\text { to send food aids }]_{\text {theme }}$ )

a sentence such as the following must be accepted

([Il Consiglio $]_{o b j}$ è autorizzato [a inviare aiuti alimentari $]_{t h e m e}$ )

([The Council $]_{o b j}$ is authorized [to send food aids $]_{\text {theme }}$ )

This is in fact obtained by means of two transformations: passivization and null-agtcompl. The first of them produces the passive form; the second one enables for the deletion of the agent complement. In case of deletion, the parser introduces a trace, that records the presence of a covert argument. Note that "to send food aids" is a clause that undergoes the same kind of processing. Consequently, "food aids" is recognized as the object of "send". The subject is deleted, but in this case it is recovered from the main clause: when its verb is "authorize", then the implicit subject of the dependent clause is equal to the object of "authorize" (the Council): this rule is called obj-equi. Note also that in the passive form, the deep function of "The council" (i.e. "object") must be (and in fact is) recovered in order to allow obj-equi to work properly also in this case. Finally, in the passive example, the indirect object of "send" is known to exist, but it is unspecified; the actual final representation is:

[Il Consiglio $]_{o b j, 1}$ è autorizzato $\left[\mathrm{t}_{u}\right]_{s u b j}\left[\mathrm{a}\right.$ inviare $\left[\mathrm{t}_{1}\right]_{s u b j}\left[\mathrm{t}_{u}\right]_{i n d o b j}$ [aiuti alimentari $\left.]_{o b j}\right]_{\text {theme }}$

([The Council $]_{o b j, 1}$ is authorized $\left[t_{u}\right]_{s u b j}$ [to send $\left[t_{1}\right]_{s u b j}\left[t_{u}\right]_{\text {indobj }}$ [food aids $\left.]_{\text {obj }}\right]_{\text {theme }}$ )

That is, "some unknown entity authorizes the Council that the Council sends to some other unknown entity food aids" (the complete dependency tree is provided in Figure 3). Such a representation is the basis for the final step of semantic interpretation, described in the next section.

\subsubsection{Punctuation and syntactic analysis}

The parser splits the input into sentences mostly based on punctuation: an undesired result is that in some cases parts of the text that should be treated as single units are 


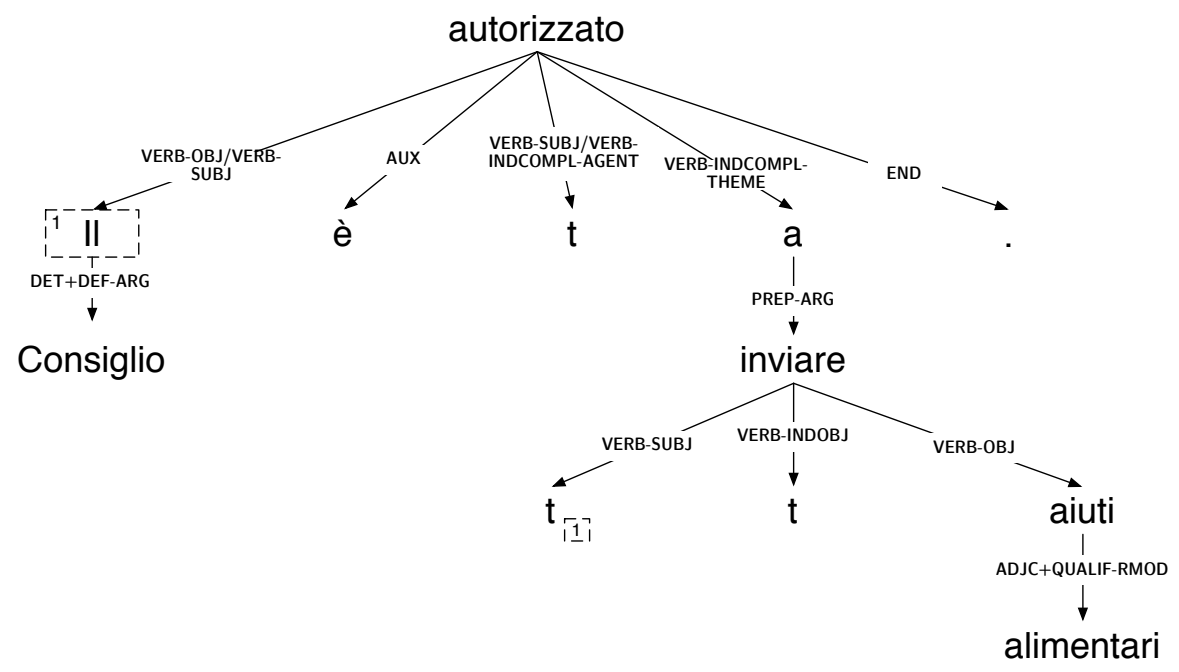

Figure 3: Full parse tree of the sentence 'Il Consiglio è autorizzato a inviare aiuti alimentari'. The dotted subscript of the trace $t_{1}$ indicates a reference to the node 1 , corresponding to the word "Il".

broken into different sub-parts. In these cases, pieces of information that need be considered as a whole result scattered in different trees.

Legal jargon uses punctuation in many complex ways, and a computational system needs to cope with these phenomena. In particular legislative texts use punctuation to "shape" the typographical structure that will constrain the discourse structure of the text. Moreover, legislative texts use punctuation to mark the syntactic structure, too. Let us consider the following sentence:

Dopo la RIF151: VIR1 sono inserite le seguenti: VIR2.

After RIF151: VIR1 are inserted the following: VIR2.

This sentence reflects a use of a colon that is proper in legal jargon: before specifying a reference to a legal source, a colon is added to add clarity and emphasis, like an actor would do by introducing a pause. Providing a computational account for such specific constraints is not trivial. Indeed, the standard behaviour of TUP for punctuation splits the sentence into three different blocks:

$$
\overbrace{\text { After RIF151: }}^{i} \overbrace{\text { VIR1 are inserted the following: }}^{i i} \overbrace{\text { VIR2 }}^{i i i} \text {. }
$$

thereby producing three different dependency trees, i.e. one for each block. This behaviour, that -for instance- fits well to newspaper texts, hides the syntactic dependencies between the words belonging to different blocks. As a consequence, the relevant information to recognize the modification is scattered over the three trees, and so the semantic interpreter (see next Section) will not be able to recognize the modification. 
One possible solution could be to modify TUP to account for specific cases of punctuation, but this solution would result in undertaking a series of ad-hoc solutions that would result in a scarcely robust approach. Another possible solution could be to modify the semantic interpreter in order to search the relevant information over a number of dependency trees. The drawback of this choice is that it simply passes the problem to the semantic interpreter, making it unnecessarily complex. The adopted solution was to design a Punctuation Manager algorithm that modifies the original punctuation. The working hypothesis underlying the algorithm is that a text fragment containing the information about a modification has a typical "shape", and we can modify the punctuation in the fragment to follow this shape. Considering a text fragment that comes from a single corpo element, the following steps are applied:

1. On the basis of the punctuation in the fragment, TUP produces a sequence of parse trees.

2. A regular expression defined on the POS tag of the roots of these trees groups together text blocks and rewrites the original punctuation (e.g., by deleting ':') so that a new fragment is produced.

3. This new fragment is parsed and the resulting trees are passed to the semantic interpreter.

We analyzed a number of corpo modifications and on the basis of empirical observation we found that the corresponding fragments correspond to one (possibly empty) fragment of text that is syntactically governed by an article or a preposition (i.e., $[P R E P \mid A R T]$ ?), followed by a block of text that is syntactically governed by a verb $\mathrm{V}$, and a number of (possibly empty) blocks of texts that are syntactically dominated by a noun, a number or an article $[\mathrm{N}|\mathrm{NUM}| \mathrm{ART}] *$. The fragment in (1), where the three partitions mentioned above are marked as $i$ ), ii) and iii), would be rewritten and grouped as follows:

$$
\overbrace{\text { After RIF151 VIR1 are inserted the following VIR2. }}^{i}
$$

This version of the Punctuation Manager algorithm relies on the assumption that an entire modification is contained in a $\langle$ corpo $\rangle$ element; however, other parts of the text, as the ones marked as $\left\langle\right.$ alinea ${ }^{16}$ in the example below, can be involved.

A decorrere dalla data di entrata in vigore del presente regolamento sono abrogati: a) la RIF135; b) il RIF137; c) RIF138.

From the date of entry into force of current regulation, the following are repealed: a) the RIF135; b) the RIF137; c) RIF138.

The input encoding for this sentence is displayed in Figure 4, where each RIF element of the list identifies a source (namely, a law and two decrees) to be repealed. In particular, only the $\langle$ alinea $\rangle$ element contains a verb: the modification cannot be discovered by only considering the $\langle$ corpo $\rangle$ : the corpo can be syntactically dominated by

${ }^{16}$ The $\langle$ alinea $\rangle$ tag is an element used in the annotation of legal texts to govern an enumeration of elements. 


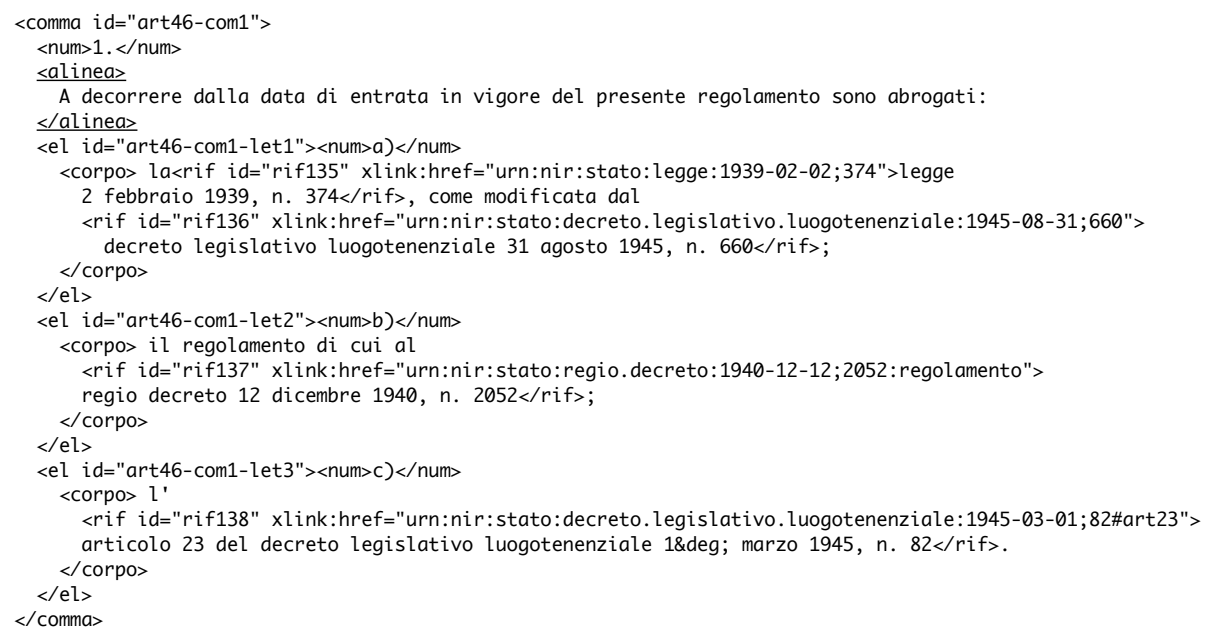

Figure 4: The structure of a alinea spans over three elements, each one including a corpo.

a noun, and the missing verb can be found in the alinea. Therefore, regardless of the XML structure, that can include further levels such as el (element letters), en (element numbers) and numbers recursively nested, we keep track of the text contained in the element alinea. In case no verb is found in the corpo fragment, no sequence of parse trees matched the regular expression, and the alinea fragment is syntactically governed by a verb, we merge the fragments dominated by the verb in the alinea in a single parse tree. Each dependency tree, that now potentially contains all the information needed to detect a modification, is then processed by the Semantic Interpreter.

\subsection{Semantic Interpretation}

The parser returns a dependency tree that represents the syntactic analysis of the source Italian sentence.

A modification is represented in terms of a semantic frame composed of slots [5], such as legalCategory, referenceDocument, modifyingText and modifiedText, as depicted in Figure 5 Retrieving a modificatory provision amounts to choosing a frame to describe the modification at hand, and to filling its slots with the appropriate arguments. This is accomplished by inspecting the verb and its dependents, and by finding a match between the frames and the syntactic and semantic information currently available. Since there can be more than one match, some heuristics are used to choose the best one.

The semantic interpreter is a rule-based system. Rules make use of two different sorts of information. The first one is the syntactic information produced in output by the parser; the second one comes from the legal provisions taxonomy (Section 4.2 ) and 
Frame substitution

\begin{tabular}{lc} 
referenceDocument & $\mathrm{S} 2603265 . \mathrm{xm}$ \\
\hline modifiedText & $\operatorname{vir}_{1}$ \\
\hline modifyingText & $\operatorname{vir}_{2}$ \\
\hline
\end{tabular}

Figure 5: Example of a frame for representing a legal modification.

Table 1: Example of tests on the verb dependents, and of the corresponding actions undertaken.

\begin{tabular}{|c|c|}
\hline \multirow{3}{*}{ IF } & $\begin{array}{l}\text { - the subtree attached to the verb by a } R M O D \text { label (that is, modifier) } \\
\text { contains a } R I F_{1} \text { constant; } \text { AND }\end{array}$ \\
\hline & $\begin{array}{l}\text { - the subtree attached to the verb by a } S U B J \text { label (that is subject) } \\
\text { contains a } V I R_{1} \text { constant; AND }\end{array}$ \\
\hline & $\begin{array}{l}\text { - the subtree attached to the verb by a } O B J \text { label (that is, object) } \\
\text { contains a } V I R_{2} \text { constant }\end{array}$ \\
\hline \multirow{3}{*}{ THEN } & - fill referenceDocument with he $R I F_{1} \mathbf{A N D}$ \\
\hline & - fill modifiedText with the $V I R_{2}$ AND \\
\hline & - fill modifyingText with the $V I R_{1}$ \\
\hline
\end{tabular}

exploits linguistic background knowledge. Such a knowledge base is used to store the information about verbs and their use, such as verb classes, mandatory and optional dependents, and their connections with the modification types.

One general rule tests whether the root node of the syntactic tree is a verb, and if it belongs to the modificatory provisions taxonomy. In this case, we have a fundamental cue that the sentence being analyzed contains a modificatory provision and -based on the taxonomy-, we are informed of the possible legalCategory of the modification. Rules are composed of a set of IF-THEN tests, that inspect the dependents of the verb to fill the slots of the current frame. For example, if we find that the root node is the verb "sostituire" (to substitute), the semantic frame associated with the legalCategory: substitution is instantiated, and a set of tests is executed on the verb dependents to fill the appropriate slots. Each legal category has its own slots, so that verbs such as substitute, change, modify, etc. have in common the same set of slots, as appropriate for the legal category substitution. In this way we can extend incrementally, and in modular fashion, each legal category through adding novel verbs.

In particular, the rules are charged to discover whether in the syntactic arguments like subject, object or in the verb modifiers any constant such as RIF or VIR is present. As we have seen in Section 5.1, such constants refer to a legal source and to an excerpt of text, respectively, so they would be natural candidates as fillers for the slots defining the position of the modificatory provision, the referenceDocument, the modifyingText and the modifiedText.

Let us introduce the rule presented in Table 1 by starting from a simple linguistic realization of a substitution modification. The verb sostituire (to replace) is usually connected with the substitution modification, so that we expect that both the modify- 


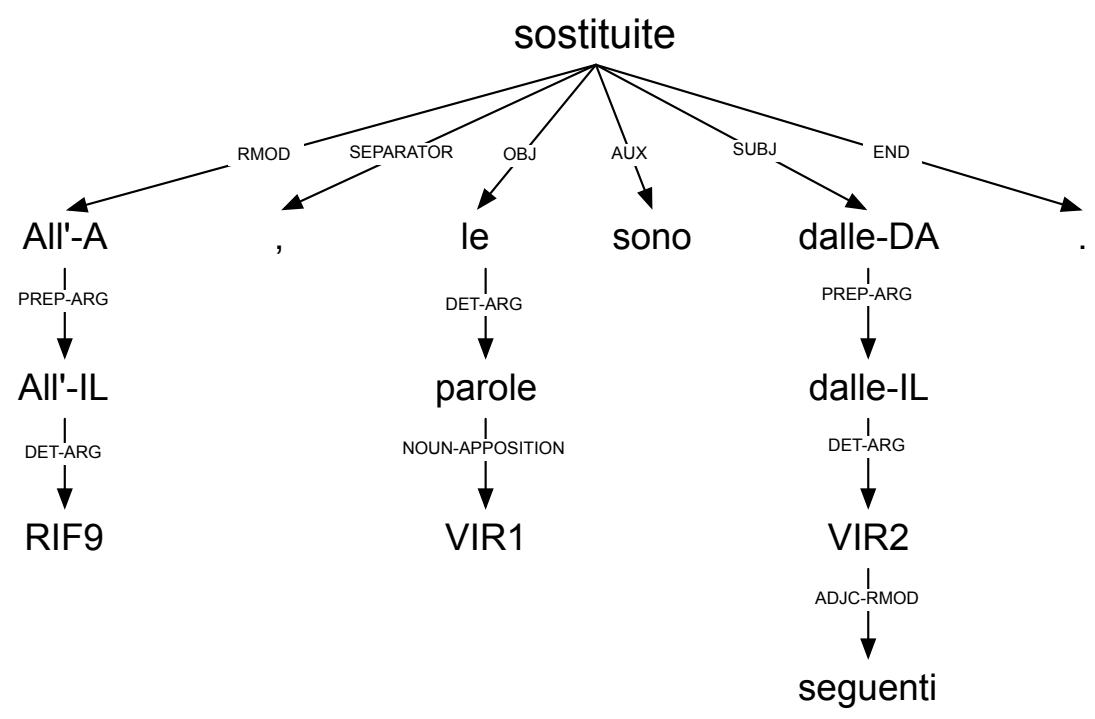

Figure 6: Syntactic analysis of the sentence: "All'RIF9, le parole VIR1 sono sostituite dalle seguenti VIR2" (At the RIF9, the words VIR1 are substituted by the following VIR2).

ingText and the modifiedText slots need to be filled (Figure 55. A sentence containing a modificatory provision could thereby have the form

The words VIR 1 substitute for the words $\mathrm{VIR}_{2}$.

Additionally, we expect that also the source document is mentioned where the modification should occur; so that a sentence containing a substitution could actually be in the form

At RIF the words VIR 1 substitute for the words $\mathrm{VIR}_{2}$.

To extract information about who substitutes for what and where, we inspect three branches of the tree, looking for the $S U B J$ (subject), the $O B J$ (object) of the sentence (to recognize the modifyingText and the modifiedText, respectively), and the $R M O D$ (restrictive modifiers) to retrieve where the modification occurs. The parser computes passivization. It means that in a sentence in passive form the agent is recognized to play the same deep role played by the subject in (the same) active sentence. Similarly, the subject in a passive sentence is recognized to play the same role of the object in (the same) active sentence. Such features of the parser allow us to connect the former role to the modifyingText and the latter one to the modifiedText, so that the same rule in Table 1 applies to the sentence

At RIF the words $\mathrm{VIR}_{2}$ are substituted with the words $\mathrm{VIR}_{1}$.

The final realization in the sentence (5) can then be analyzed through the mentioned rule, and the modification frame is filled. 
Further rules are designed to account for complex linguistic constructions, such as the case of coordination. Let us consider the following sentence: All'articolo 1, comma 2, sono soppresse le lettere d) edf); (At article 1, comma 2, the letters d) andf) are deleted;). It is converted into: All'RIF16, sono soppresse le RIF34) e RIF35), (At RIF16, the RIF34 and RIF35 are deleted). TUP recognizes coordination and marks it with the COORD relation. The semantic frame corresponding to the deletion of RIF34 is filled as in the case of substitution (the main difference being that the slot modifyingText is not present); additionally, the semantic interpreter recognizes the presence of a coordinate sentence, since the reference RIF34 has a descendant node RIF35 in a subtree that is connected by the COORD labeled edge. In this case the semantic interpreter produces two distinct frames, one for each coordinate.

\section{Experimentation and Evaluation}

The goal of our research is to build software tools to assist human annotators in identifying modificatory provisions and qualifying them with as many details as possible following the legal taxonomy of NIR. To assess our work, we started from a set of 1540 files NIR DTD 2.0 valid, containing overall 4,635 modifications. In this dataset the three modification categories (or types) integration, substitution and repeal are by far the most common types, as Table 2 shows; namely, 3,285 modificatory provisions fall into one of such these classes, thereby covering $70.87 \%$ of the grand total.

\subsection{Experimental Setting}

In current experiment we focussed on documents organized in articles, called articolati; we considered only textual modificatory provisions of either the integration, substitution or deletion type. This resulted in considering 177 files, for a total amount of 11,855 XML corpo elements (textual part of the article), and an overall amount of 2, 206 modificatory provisions (namely, 772 integrations, 843 substitutions and 591 deletions ${ }^{17}$ hand-qualified by the High Court of Cassation legal experts [13] using Norma-Editor [28], which were considered for the experimentation.

The goal of the experiment is twofold, since it concerns both the utility of the tool as an assistant to human annotators, and as a completely automatic annotation tool. Accordingly, two accuracy measures were devised.

- Measure A. The percentage of modificatory provisions correctly annotated with the pair 〈legal category, position〉, where legal category is one in \{integration, substitution, deletion\}, and position is the constant identifying the file and the position inside the file where the modification occurs;

- Measure B. The percentage of modificatory provisions correctly annotated with the tuple $\langle$ type, position, novella, novellando $\rangle$, where type and position are the same as in Measure A, and novella and novellando are both excerpts of quoted

${ }^{17}$ The dataset is available for download at the URL: http://www.di.unito.it/ radicion/AI_LAW_2012/ 
Table 2: Number of modifications occurring more than 50 times in the dataset, showing that $70.87 \%$ of the modifications are of either integration, substitution or deletion type.

\begin{tabular}{r|r} 
modif. type & $\sharp$ of modif. \\
\hline substitution & 1,368 \\
integration & 1,186 \\
repeal & 731 \\
variations & 405 \\
derogation & 303 \\
range & 136 \\
modification of terms & 133 \\
validity & 61 \\
execution (attuazione) & 59 \\
others & 253 \\
\hline Total & 4,635
\end{tabular}

Table 3: The accuracy obtained on each type of modificatory provisions.

\begin{tabular}{|r|c|c|c|c|}
\hline Measure A & integration & substitution & repeal & total \\
\hline precision & $99.46 \%$ & $99.87 \%$ & $93.68 \%$ & $98.35 \%$ \\
\hline recall & $95.47 \%$ & $90.75 \%$ & $67.68 \%$ & $86.22 \%$ \\
\hline
\end{tabular}

\begin{tabular}{|r|c|c|c|c|}
\hline Measure B & integration & substitution & repeal & total \\
\hline precision & $94.33 \%$ & $94.26 \%$ & $90.87 \%$ & $93.54 \%$ \\
\hline recall & $90.54 \%$ & $85.65 \%$ & $65.65 \%$ & $82.00 \%$ \\
\hline
\end{tabular}

text. The novella (modifyingText in the above notation) is the portion of text being added, whilst novellando (modifiedText in the above notation) is the portion of text being modified. Either the novella or the novellando -or both- may be absent. E.g., deletions usually lack both novella and novellando, while in integrations we usually do not have the novellando. The measure B scores 1 point in case all elements of the tuple are correctly identified; 0 in case anyone of them is wrong.

\subsection{Results and discussion}

Our system obtained $98.3 \%$ precision and $86.2 \%$ recall, computed with the Measure $A$; and $93.5 \%$ precision and $82.0 \%$ recall computed according to the Measure $B$. More details on results are provided in Table 3 , where the recall and the precision for both measures on the specific types of modificatory provisions are reported, and in Appendix A providing analytical results on each and every file in the dataset. Such results qualify the system as a robust one, still in need of improvements, but closer to being actually used by legal drafters and law scholars.

We are testing a rather complex architecture that involves software modules $i$ ) to extract the meaningful parts of the documents (Section 5.1); ii) to parse them (Section 5.2); and iii) to identify and annotate legal modifications (Section 5.3). There- 
Table 4: Accuracy of the TULSI system on individual files: the recall on repeals decreases as the number of repeals per file increases.

\begin{tabular}{r|c|c|c|c} 
Repeals per file & 1 & 2 to 5 & 6 to 10 & more than 10 \\
\hline Recall & $77.14 \%$ & $64.76 \%$ & $65.38 \%$ & $62.67 \%$
\end{tabular}

fore, it is worth trying to disaggregate the results of such components, and to focus on the results provided in the semantic annotation process alone. In this way, we can compare the modifications extracted by TULSI with the modifications that have been correctly parsed. The rationale is that in the first two steps some excerpts of legal texts are skipped, thereby undermining the performance of the semantic annotation phase. In the dataset there were 11,855 〈corpo $\rangle$ elements, 11,080 of which were correctly parsed, and 775 were skipped. This is interesting, in that it says that some $6.4 \%$ of all possible texts containing modifications are lost before the semantic annotation phase. However, this datum suggests that improvements on the input preprocessing routines and a rigorous check of the annotated files are still possible. In order to shed light on the accuracy of the semantic interpreter, we define the upper-bound recall measure as the percentage of correctly identified modificatory provisions on the number of modificatory provisions that are actually analyzed by the parser. If we consider the upper-bound recall computed with the Measure B, the system's accuracy raises to $84.8 \%$.

Before providing the analysis of various sorts of errors (Section 6.2.2), we report the results of another experimentation on a baseline against which we compare the figures obtained by TULSI.

\subsubsection{Definition of a Baseline}

The rationale underlying the design of the baseline was to provide an estimate of the difficulty of the task, and to investigate in how far linguistic information affects the accuracy of the system, by limiting the contribution of linguistic information. So we maintained the same preprocessing procedures described in Section 5.1. That is, the new system performs the same XML preprocessing to extract the corpo elements; also we designed some simple regular expressions that accept "RIF" and "VIR" occurrences. The underlying assumption is that the whole information is contained in the corpo elements, which we know beforehand to be a rough approximation. In particular the new system does not handle cases where the main verb is contained in an alinea element and its objects in the corpo elements, since this strategy would require syntactic analysis. Moreover, we used a simpler lemmatizer for Italian [41], different from that included in the TULSI system; in particular the lemmatizer does not make use of POS tagging nor morphological information.

The core of the baseline system implements a simple string-based pattern matching algorithm that takes into account the standard sentence word order for Italian, that is subject-verb-object $(S V O)$ [4]. The algorithm recognizes the presence of a meaningful verb (similar to the TULSI system), and then it inspects the surrounding words in order to retrieve the modificatory provision arguments. To these ends, a tenth of regular expressions were implemented. For example, in the sentence RIF21 is repealed the 
Table 5: The accuracy obtained by the baseline system on each type of modificatory provisions.

\begin{tabular}{|}
$\mid$ Measure A & integration & substitution & repeal & total \\
\hline precision & $70.79 \%$ & $77.28 \%$ & $64.03 \%$ & $72.15 \%$ \\
\hline recall & $90.41 \%$ & $93.59 \%$ & $52.11 \%$ & $81.37 \%$ \\
\hline Measure B & integration & substitution & repeal & total \\
\hline precision & $20.49 \%$ & $42.90 \%$ & $30.35 \%$ & $31.59 \%$ \\
\hline recall & $26.17 \%$ & $51.96 \%$ & $24.70 \%$ & $35.63 \%$ \\
\hline
\end{tabular}

baseline system is able to recognize that RIF21 is the argument of the repeal. This simple approach does not allow to properly recognize permutations of arguments that occur in more complex syntactic constructions, e.g., passive sentences.

The baseline system has been tested on the same dataset, and it obtained the figures reported in Table 5 The results on the Measure $A$ are rather similar to those of the TULSI system; they confirm that a simple occurrence of a meaningful verb is a strong cue about the presence of a modificatory provision. Although the precision is significantly lower than that obtained by TULSI, the recall is high (in the case of $s u b$ stitutions the baseline system obtains am higher score than the TULSI system). The Measure $B$ proves that the problem is not a trivial one, since a naive approach based on a simple pattern-matching turns out to be not sufficient to account for the possible arguments permutations that are typical of Italian syntax.

\subsubsection{Analysis of errors}

Before analysing the main causes of failures, we elaborate on the possible reasons of the poor accuracy obtained in extracting repeals. Based on an analysis of the repeals that were not correctly analyzed, we observe that one major reason lies in the preprocessing stage, where the handling of the corpo elements and in particular of the alinea elements needs further refinements. One paradigmatic example of such issues is the following case, where an alinea governs many repeals. The text contained in the alinea is: 'From the date of entry into force of the current decree, the following provisions are repealed: [...]'. The alinea spans over further corpo elements, so that the system generates the following excerpts (see Section 5.2.1) that are parsed and analysed in search of modificatory provisions:

1. $\lfloor$ From the date of entry into force of current decree, the following provisions are repealed $\rfloor_{\text {alinea }}\lfloor\text { the RIF93, and related enclosed norms }\rfloor_{\text {corpo; }}$;

2. [From the date of entry into force of current decree, the following provisions are repealed $\rfloor_{\text {alinea }}\lfloor$ the RIF129, published in the appendix [...], containing norms about the maritime transport of dangerous goods $[\ldots]\rfloor_{\text {corpo }}$;

3. ...

In this case, the nominal structure of the corpo number 1. meets our assumptions that the alinea contains the verb governing the objects present in the corpo elements 
(please refer to Section 5.1 and to Figure 4). In contrast, in case 2. the governor of the corpo is not a noun. As a result, the Punctuation Manager algorithm correctly extracts only the modification in the case 1 ., and we lose overall 16 repeals out of the 18 present in the considered file 18

Since repeals often occur in this form, with an alinea and a number of corpo elements, the difficulty in handling this sort of linguistic structures explains the lower recall on repeals. This intuition is also confirmed by the data in Table 4 , when many repeals are present in a single file, the sentence structure is more complex and the recall decreases substantially.

\section{On preprocessing and annotation errors}

Although some annotations errors were corrected in the preprocessing stage, some of them were still present in the input texts. Some spot errors in the automatic annotation can be useful to provide a feedback to the activity of human annotators. Also, some annotation errors can be used to draw some observations about how the elements in the NIR DTD are actually designed and about how they should be used in principle.

One major source of confusion is about what should be included in the element RIF, and what should not or what is the source of misinterpretations by the parser. Here in the following we briefly illustrate a (non exhaustive) list of usages of such element that we found not compliant with its expected or regular usage.

The first sort of tagging misusage consists of cases where some text is mistakenly left out of the RIF element. Such a problem occurs when in a given sentence the same words are somewhere tagged as an RIF element and somewhere apparently inconsistently not tagged as an RIF. Let us consider the following sentence: 19

Le tabelle A e B allegate al decreto legislativo n. 533 del 1993 sono sostituite dalle tabelle A e B di cui all'allegato 2.

$\lfloor$ Tables $A\rfloor$ and $\lfloor B\rfloor$ enclosed to the decree [...] are substituted by tables $A$ and $B$ of current law.

In this case the sentence is rewritten into:

RIF108 and RIF286 [. . .] are substituted by tables A and B of RIF287.

The words 'tables A and B' in the following sentence are subject to a different treatment: in particular, the second occurrence of 'tables A and B' has not been annotated by human experts as an RIF because it represents a characterization of the modification that strongly relies on the annex (RIF287). Therefore it is not possible for the parser to build the correct syntactic structure.

A completely different sort of error occurred in cases where the RIF element is also the subject of the sentence, such as in the following: 20

At $\lfloor$ Article 1 of the decree [...], comma 5」 is substituted by [...].

18 http://www.di.unito.it/ radicion/AI_LAW_2012/S2504725.xml

19 http://wWw.di.unito.it/ radicion/AI_LAW_2012/S2507927.xml

2c/http://wWw.di.unito.it/ radicion/AI_LAW_2012/S2503953.xml 
the sentence is rewritten into

\section{At RIF73 is substituted by [...].}

Since the preposition 'at' is out of the RIF73, the parser wrongly analyzes it as a modifier, whilst it is the subject of the original sentence. The annotation guidelines prescribe that prepositions are not to be included within RIF constants, however the word 'comma' -which is the subject of the sentence- should not be hidden in the constant RIF that is associated with the prepositional group. In Section 5.1 we have considered the case in which a preposition was wrongly included within the RIF elements; in these cases it is still possible to resort to the correct preposition by inspecting the text substituted through the RIF constant and taking out of it such prepositions. Now, a similar problem occurs when the verb of the sentence is improperly included within the RIF element, but in such cases there is no easy way out. One typical such error determines the following rewriting ${ }^{21}$

The $\lfloor$ title is substituted $\rfloor$ by the following \implementation of the directives $\rfloor$. The RIF115 by the following VIRI.

Still different issues arise when a verb usually associated with a given type of modification is present, but the annotated type is surprisingly not coherent with the considered modification. For example, in the sentence 'the present decree with the following RIF57 modifies and substitutes the RIF58 22 both verbs are usually associated with modifications of type substitution; however, the modification has been annotated as a repeal. In similar cases we found that an integration has been confused with a substitution ${ }^{23}$ A slightly different issue arises when a sequence of two modifications is described, like in the following sentence: 24

RIF103 substitutes for RIF102 [...], which is repealed from the date of entry to force of current regulation.

Since the RIF102 substitutes for RIF103, we have a substitution detection, and not a repeal as it was annotated.

In other cases some slots of the frame have not been annotated (like the modifyingText or novella) ${ }^{25}$ or the whole modification has been skipped because the modification is not a simply textual one, but it involves a more complex human interpretation (incomplete modification tagged with the proper attribute dsp:incompleta) ${ }^{26}$ In the last case, correctly identifying the modification affected the precision but not the recall of the system's results.

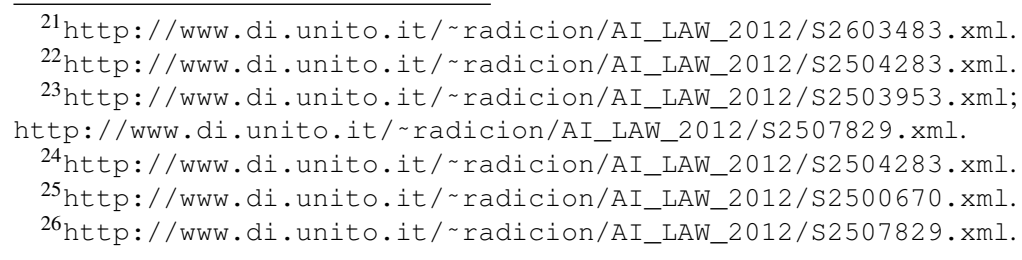


Table 6: Statistics reporting the length of parsed sentences.

\begin{tabular}{c|c|c|c|c|c|c} 
length in words & $1-10$ & $11-20$ & $21-30$ & $31-40$ & $41-50$ & $>50$ \\
\hline$\%$ of sentences & $12 \%$ & $26 \%$ & $22 \%$ & $16 \%$ & $10 \%$ & $14 \%$
\end{tabular}

\section{Parsing errors}

Most errors occurred in the parsing process. They were mainly due to complex syntactic structures whose associated difficulties are largely known in literature: e.g., in handling coordination, punctuation, relative clauses, PP-analysis ${ }^{27}$ etc..

A first observation is about the length of parsed sentences. It is in general largely acknowledged that the difficulty inherent in parsing increases in non linear fashion with the length of the input sentence. For example, in contests for Italian parsers, it is customary to disregard sentences longer than 40 words [12]. To these ends it is interesting to notice that in the dataset analysed (i.e., after the substitution of RIF and VIR elements) the average sentence length is 29.9 words, with the distribution illustrated in Table 6 In particular, it is noteworthy that about $25 \%$ of parsed sentences were longer than 40 words, corresponding to 3,164 sentences out of the 13,144 sentences extracted from the $\langle$ corpo $\rangle$ elements.

Finally, specialized words from the legal jargon caused different sorts of errors, along with misspelled words like 'irserito' in place of 'inserito' (inserted) ${ }^{28}$

\section{Interpreter rules}

An unexpected outcome of the present work is that the improvement with respect to past experimentation was mostly due to a careful work on the preprocessing phase and to the initial filtering, aimed at providing the system with a robust input, while the impact of the refinements of the semantic interpreter rules was limited.

The experimentation showed that to obtain high accuracy it is fundamental to have a clean input, and that a compact set of rules (around 25, also including those handling coordination) is enough to identify modifications in a large collection of documents. Even though Occam's razor has been subject to severe criticism in fields such as machine learning [19], for rule-based systems the principle that given two models with the same error, the simpler one should be preferred because simplicity is desirable in itself is still valid for two reasons. First, writing rules is a laborious activity, and sets of rules need to be maintained over time; so a slimmer set of rules will be maintained more easily than a large one. Second, we expect that the same few rules that describe 2,206 modificatory provisions will work -all other elements being equal- similarly on a new dataset.

\footnotetext{
${ }^{27}$ E.g., a sentence like 'dalla data del RIF32, il RIF33 è abrogato' (from the date of RIF32, RIF33 is repealed) is ambiguous in Italian, and the parser wrongly accounts for the dependent 'dalla data' -translated into 'from the date' - with the agent of a passive action, which is introduced by the same preposition. 


\section{Conclusions and Future Works}

We have introduced the relevant problem of the automatic annotation of legal provisions, involving several challenging open issues. In particular, current research has a twofold interest: on the theoretical side, we showed that a hybrid approach coupling deep syntactic parsing and shallow semantic interpretation leads to satisfactory results. On the other hand, from a practical perspective, we delivered a robust NLP system that can speed-up the process of semantic metadata annotation of legal documents containing modificatory provisions.

We have recalled the NIR representation adopted for the both legal texts and modificatory provisions; we have introduced the taxonomy of legal modifications and the corresponding metadata format that is used throughout the process of annotation of modificatory provisions. We then have illustrated the extraction of modificatory provisions as a three-step process, where we first retrieve the relevant excerpts of text, parse them, and map the resulting parse trees onto the appropriate semantic frames. We have described a hand-crafted dataset used as a gold-standard for experimenting with the implemented system; we have then discussed the results obtained and reviewed some interesting types of errors, pointing out possible improvements for the future. Finally, we have compared the performance of the TULSI system against a baseline system, specifically developed to estimate the complexity of the task and to investigate how much syntactic analysis contributes to solving the problem.

To summarize, considering the difficulty of the task and the noise present in data, our results are notable. We have illustrated how a systematic error analysis can produce feedbacks beneficial to annotators, both to correct spot errors and to revise annotation practices. The final precision $93.6 \%$ makes the system appropriate to assist human annotators in quickly identifying modificatory provisions, and sometimes (namely, in $81.6 \%$ of cases) to fully qualify them. On the other side, much work is still necessary to enhance the accuracy in the particular case of repeals, that are linguistically harder. Some work is also required to improve the preprocessing routines to extract and to conveniently filter the input, and to extend the coverage to other types of modificatory provisions.

One final remark is about the evaluation of the work in the perspective of future improvements of the system: the principal contribution of this work is to focus on some individual steps of the linguistic analysis. We implemented a full computational linguistics analysis pipeline that allowed us to weigh the contribution of each level of linguistic analysis. Based on experimental evidence, we realized that the semantic interpretation is simple, and it can be accounted for by a reduced number of semantic rules. In contrast, the final performance of the system heavily relies on the preprocessing phase, and on the syntactic analysis. 


\section{References}

[1] Steven P. Abney. Principle-Based Parsing: Computation and Psycholinguistics, chapter Parsing by Chunks. Kluwer, Dordrecht, 1991.

[2] AIPA. Formato per la rappresentazione elettronica dei provvedimenti normativi tramite il linguaggio di marcatura XML. Circolare n. AIPA/CR/40, 22 aprile 2002.

[3] Carlos E. Alchourròn and Eugenio Bulygin. Normativity and Norms: Critical Perspectives on Kelsenian Themes, chapter The Expressive Conception of Norms. Clarendon Press, Oxford, 1971.

[4] Anita Alicante, Cristina Bosco, Anna Corazza, and Alberto Lavelli. A treebankbased study on the influence of Italian word order on parsing performance. In Nicoletta Calzolari (Conference Chair), Khalid Choukri, Thierry Declerck, Mehmet Uğur Doğan, Bente Maegaard, Joseph Mariani, Jan Odijk, and Stelios Piperidis, editors, Proceedings of the Eight International Conference on Language Resources and Evaluation (LREC'12), Istanbul, Turkey, may 2012. European Language Resources Association (ELRA).

[5] Douglas E. Appelt and David J. Israel. Introduction to information extraction technology. Tutorial at IJCAI-99, 1999.

[6] Timothy Arnold-Moore. Automatically processing amendments to legislation. In ICAIL, pages 297-306, 1995.

[7] Timothy Arnold-Moore. Automatic generation of amendment legislation. In Proceedings of the International Conference on Artificial Intelligence and Law (ICAIL), pages 56-62, 1997.

[8] Roberto Bartolini, Alessandro Lenci, Simonetta Montemagni, Vito Pirrelli, and Claudia Soria. Semantic mark-up of italian legal texts through nlp-based techniques. In Proceedings of LREC 2004, pages 795-798, 2004.

[9] Carlo Biagioli, Enrico Francesconi, Andrea Passerini, Simonetta Montemagni, and Claudia Soria. Automatic semantics extraction in law documents. In ICAIL '05: Proceedings of the 10th international conference on Artificial intelligence and law, pages 133-140, New York, NY, USA, 2005. ACM.

[10] Carlo Biagioli, Enrico Francesconi, PierLuigi Spinosa, and M. Taddei. The NIR project: Standards and tools for legislative drafting and legal document web publication. In Proceedings of ICAIL Workshop on e-Government:Modelling Norms and Concepts as Key Issues, pages 69-78, 2003.

[11] Andrea Bolioli, Luca Dini, Pietro Mercatali, and Francesco Romano. For the Automated Mark-up of Italian Legislative Texts in XML. In T. Bench-Capon, A. Daskalopulu, and R. Winkels, editors, Legal Knowledge and Information Systems. Proceedings of Jurix 2002: The Fifteenth Annual Conference. IOS Press, 2002. 
[12] Cristina Bosco, Simonetta Montemagni, Alessandro Mazzei, Vincenzo Lombardo, Felice Dell'Orletta, and Alessandro Lenci. Evalita'09 parsing task: comparing dependency parsers and treebanks. In Proceedings of Evalita'09, Reggio Emilia, Italy, 2009.

[13] Raffaella Brighi and Monica Palmirani. Legal Text Analysis of the Modification Provisions: a Pattern Oriented Approach. In Proceedings of the International Conference on Artificial Intelligence and Law (ICAIL), 2009.

[14] Manola Cherubini, Gerardo Giardiello, Simone Marchi, Simonetta Montemagni, PierLuigi Spinosa, and Giulia Venturi. NLP-based metadata annotation of textual amendments. In Proceedings of Workshop on Legislative XML 2008, Jurix, 2008.

[15] Michael Collins. Three generative, lexicalised models for statistical parsing. In In Proceedings of the 35th Annual Meeting of the Association for Computational Linguistics, pages 16-23, 1997.

[16] Emile de Maat, Kai Krabben, and Radboud Winkels. Machine learning versus knowledge based classification of legal texts. In IOS Press, editor, In Proceeding of the 2010 conference on Legal Knowledge and Information Systems: JURIX 2010: The Twenty-Third Annual Conference, pages 87-96, Amsterdam, 2010.

[17] Emile de Maat, Radboud Winkels, and Tom M. van Engers. Automated Detection of Reference Structures in Law. In Tom M. van Engers, editor, Proceedings of the JURIX 2006 on Legal Knowledge and Information Systems: The Nineteenth Annual Conference, pages 41-50, Amsterdam, 2006. IOS Press.

[18] Rodrigo De Salvo Braz, Roxana Girju, Vasin Punyakanok, Dan Roth, and Mark Sammons. An inference model for semantic entailment in natural language. In AAAI'05: Proceedings of the 20th nat. conf. on Artificial intelligence, pages 1043-1049. AAAI Press, 2005.

[19] Pedro Domingos. The Role of Occam's Razor in Knowledge Discovery. Data Mining and Knowledge Discovery, 3:409-425, 1999.

[20] Aria D. Haghighi, Andrew Y. Ng, and Christopher D. Manning. Robust textual inference via graph matching. In HLT '05: Proceedings of the conf. on Human Language Technology and Empirical Methods in NLP, pages 387-394, Morristown, NJ, USA, 2005. ACL.

[21] Peter Jackson and Isabelle Moulinier. Natural language processing for online applications. Text retrieval, extraction and categorization, volume 5 of Natural Language Processing. Benjamins, Amsterdam, Philadelphia, 2002.

[22] Leonardo Lesmo. The rule-based parser of the nlp group of the university of torino. Intelligenza Artificiale, 2(4):46-47, June 2007.

[23] Leonardo Lesmo and Vincenzo Lombardo. Transformed Subcategorization Frames in Chunk Parsing. In Proceedings of the 3rd International Conference on Language Resources and Evaluation (LREC 2002), pages 512-519, Las Palmas, 2002. 
[24] Caterina Lupo, Fabio Vitali, Enrico Francesconi, Monica Palmirani, Radboud Winkels, Emile de Maat, Alexander Boer, and Paolo Mascellani. General XML format(s) for legal Sources - ESTRELLA European Project. Deliverable 3.1, Faculty of Law, University of Amsterdam, Amsterdam, The Netherlands, 2007.

[25] L. T. McCarty. Deep semantic interpretations of legal texts. In ICAIL '07: Proceedings of the 11th international conference on Artificial intelligence and law, pages 217-224, New York, NY, USA, 2007. ACM.

[26] Yasuhiro Ogawa, Shintaro Inagaki, and Katsuhiko Toyama. Automatic consolidation of japanese statutes based on formalization of amendment sentences. In Proceedings of the 2007 conference on New frontiers in artificial intelligence, JSAI'07, pages 363-376, Berlin, Heidelberg, 2008. Springer-Verlag.

[27] Monica Palmirani. Legislative XML for the Semantic Web, chapter Legislative Change Management with Akoma-Ntoso, in Legislative XML for the Semantic Web. Springer, Berlin / Heidelberg, 2011.

[28] Monica Palmirani and Federica Benigni. Norma-System: A Legal Information System for Managing Time. In Carlo Biagioli, Enrico Francesconi, and Giovanni Sartor, editors, Proceedings of the V Legislative XML Workshop, pages 205-223. European Press Academic Publishing, February 2007.

[29] Monica Palmirani and Raffaella Brighi. An XML Editor for Legal Information Management. In R. Traunmüller, editor, Electronic Government, volume 2739 of LNCS, pages 421-429, Berlin / Heidelberg, 2003. Springer-Verlag.

[30] Monica Palmirani and Raffaella Brighi. Time model for managing the dynamic of normative system. Electronic Government, pages 207-218, 2006.

[31] Monica Palmirani and Raffaella Brighi. Model Regularity of Legal Language in Active Modifications. In Biasiotti M. et. al., editor, AICOL Workshops 2009, pages 54-73, Berlin / Heidelberg, 2010. Springer.

[32] Monica Palmirani, Raffaella Brighi, and Matteo Massini. Processing Normative References on the Basis of Natural Language Questions. In DEXA '04 Proceedings of the Database and Expert Systems Applications, 15th International Workshop, pages 9-12. IEEE Computer Society, 2004.

[33] Stefano Rodotà. Clausole e principi generali nell'argomentazione giurisprudenziale degli anni novanta, chapter La tecnica legislativa per clausole generali in Italia. Cedam, Padova, 1998.

[34] Rodolfo Sacco. Lingua e diritto. Ars Interpretandi, 5, 2000.

[35] Maria Teresa Sagri and Daniela Tiscornia. Le peculiarità del linguaggio giuridico. Problemi e prospettive nel contesto multilingue europeo. mediAzioni, 7, 2009.

[36] José Saias and Paulo Quaresma. A Methodology to Create Legal Ontologies in a Logic Programming Based Web Information Retrieval System. Artificial Intelligence and Law, 12(4):397-417, 2004. 
[37] Giovanni Sartor. Il procedimento normativo regionale, chapter Riferimenti normativi e dinamica dei nessi normativi. Cedam, 1996.

[38] Claudia Soria, Roberto Bartolini, Alessandro Lenci, Simonetta Montemagni, and Vito Pirrelli. Automatic Extraction of Semantics in Law Documents. In C. Biagioli, E. Francesconi, and G. Sartor, editors, Proceedings of the V Legislative XML Workshop, pages 253-266. European Press Academic Publishing, 2007.

[39] PierLuigi Spinosa, Gerardo Giardiello, Manola Cherubini, Simone Marchi, Giulia Venturi, and Simonetta Montemagni. Nlp-based metadata extraction for legal text consolidation. In Proceedings of the 12th International Conference on Artificial Intelligence and Law, ICAIL '09, pages 40-49, New York, NY, USA, 2009. ACM.

[40] Adam Wyner. Towards annotating and extracting textual legal case elements. In E. Francesconi, editor, Informatica e Diritto: Special Issue on Legal Ontologies and Artificial Intelligent Techniques, 19(1-2), pages 9-18. ESI, 2011.

[41] Eros Zanchetta and Marco Baroni. Morph-it! A free corpus-based morphological resource for the Italian language. Corpus Linguistics 2005, 1(1), 2005. 


\section{A Appendix}

\begin{tabular}{|c|c|c|c|c|}
\hline File name & Precision (A) & Recall (A) & Precision (B) & Recall (B) \\
\hline allegato2.xml & 0.00 & 0.00 & 0.00 & 0.00 \\
\hline S2500032.xml & 1.00 & 1.00 & 1.00 & 1.00 \\
\hline S2500146.xml & 1.00 & 1.00 & 1.00 & 1.00 \\
\hline S2500167.xml & 0.00 & 0.00 & 0.00 & 0.00 \\
\hline S2500364.xml & 1.00 & 0.20 & 1.00 & 0.20 \\
\hline S2500462.xml & 1.00 & 1.00 & 1.00 & 1.00 \\
\hline S2500608.xml & 1.00 & 1.00 & 1.00 & 1.00 \\
\hline S2500625.xml & 1.00 & 1.00 & 1.00 & 1.00 \\
\hline S2500626.xml & 1.00 & 1.00 & 1.00 & 1.00 \\
\hline S2500670.xml & 1.00 & 0.91 & 1.00 & 0.91 \\
\hline S2500694.xml & 1.00 & 0.60 & 1.00 & 0.60 \\
\hline S2500745.xml & 1.00 & 1.00 & 1.00 & 1.00 \\
\hline S2500746.xml & 1.00 & 1.00 & 1.00 & 1.00 \\
\hline S2500763.xml & 0.00 & 0.00 & 0.00 & 0.00 \\
\hline S2500947.xml & 1.00 & 0.99 & 0.99 & 0.98 \\
\hline S2501052.xml & 1.00 & 1.00 & 1.00 & 1.00 \\
\hline S2501053.xml & 1.00 & 0.83 & 1.00 & 0.83 \\
\hline S2501173.xml & 1.00 & 0.97 & 0.99 & 0.96 \\
\hline S2501174.xml & 1.00 & 1.00 & 1.00 & 1.00 \\
\hline S2501206.xml & 1.00 & 1.00 & 1.00 & 1.00 \\
\hline S2501406.xml & 1.00 & 1.00 & 0.92 & 0.92 \\
\hline S2501420.xml & 1.00 & 1.00 & 1.00 & 1.00 \\
\hline S2501421.xml & 1.00 & 1.00 & 1.00 & 1.00 \\
\hline S2501517.xml & 0.97 & 0.85 & 0.94 & 0.82 \\
\hline S2501647.xml & 1.00 & 1.00 & 1.00 & 1.00 \\
\hline S2501818.xml & 0.97 & 0.94 & 0.97 & 0.94 \\
\hline S2501916.xml & 1.00 & 1.00 & 0.92 & 0.92 \\
\hline S2501939.xml & 1.00 & 1.00 & 1.00 & 1.00 \\
\hline S2502223.xml & 1.00 & 1.00 & 1.00 & 1.00 \\
\hline S2502279.xml & 1.00 & 1.00 & 1.00 & 1.00 \\
\hline S2502438.xml & 1.00 & 1.00 & 0.67 & 0.67 \\
\hline S2502496.xml & 1.00 & 0.80 & 1.00 & 0.80 \\
\hline s2502514.xml & 1.00 & 1.00 & 0.75 & 0.75 \\
\hline S2502555.xml & 1.00 & 1.00 & 1.00 & 1.00 \\
\hline S2502604.xml & 1.00 & 1.00 & 0.91 & 0.91 \\
\hline S2502698.xml & 1.00 & 1.00 & 1.00 & 1.00 \\
\hline S2502792.xml & 1.00 & 1.00 & 1.00 & 1.00 \\
\hline S2502832.xml & 1.00 & 0.98 & 0.93 & 0.91 \\
\hline S2502834.xml & 1.00 & 1.00 & 1.00 & 1.00 \\
\hline S2502836.xml & 1.00 & 1.00 & 1.00 & 1.00 \\
\hline S2503160.xml & 1.00 & 1.00 & 1.00 & 1.00 \\
\hline S2503178.xml & 1.00 & 1.00 & 1.00 & 1.00 \\
\hline S2503308.xml & 1.00 & 1.00 & 1.00 & 1.00 \\
\hline S2503365.xml & 0.86 & 0.67 & 0.86 & 0.67 \\
\hline S2503748.xml & 0.00 & 0.00 & 0.00 & 0.00 \\
\hline S2503828.xml & 1.00 & 1.00 & 1.00 & 1.00 \\
\hline S2503855.xml & 1.00 & 0.97 & 1.00 & 0.97 \\
\hline S2503917.xml & 1.00 & 1.00 & 1.00 & 1.00 \\
\hline S2503924.xml & 1.00 & 1.00 & 1.00 & 1.00 \\
\hline S2503953.xml & 1.00 & 0.95 & 1.00 & 0.95 \\
\hline S2503954.xml & 1.00 & 1.00 & 1.00 & 1.00 \\
\hline S2503999.xml & 0.00 & 0.00 & 0.00 & 0.00 \\
\hline S2504007.xml & 1.00 & 1.00 & 1.00 & 1.00 \\
\hline S2504013.xml & 1.00 & 1.00 & 1.00 & 1.00 \\
\hline S2504167.xml & 1.00 & 0.50 & 1.00 & 0.50 \\
\hline S2504172.xml & 0.50 & 1.00 & 0.50 & 1.00 \\
\hline S2504207.xml & 1.00 & 0.88 & 1.00 & 0.88 \\
\hline S2504238.xml & 0.00 & 0.00 & 0.00 & 0.00 \\
\hline S2504266.xml & 1.00 & 0.81 & 1.00 & 0.81 \\
\hline S2504283.xml & 0.00 & 0.00 & 0.00 & 0.00 \\
\hline
\end{tabular}




\begin{tabular}{|c|c|c|c|c|}
\hline S2504293.xml & 1.00 & 0.90 & 1.00 & 0.90 \\
\hline S2504345.xml & 1.00 & 0.80 & 0.00 & 0.00 \\
\hline S2504425.xml & 1.00 & 1.00 & 1.00 & 1.00 \\
\hline S2504457.xml & 1.00 & 0.95 & 0.83 & 0.79 \\
\hline S2504508.xml & 1.00 & 1.00 & 1.00 & 1.00 \\
\hline S2504542.xml & 1.00 & 1.00 & 1.00 & 1.00 \\
\hline s2504646.xml & 1.00 & 0.50 & 1.00 & 0.50 \\
\hline S2504647.xml & 1.00 & 1.00 & 0.00 & 0.00 \\
\hline S2504687.xml & 1.00 & 1.00 & 1.00 & 1.00 \\
\hline S2504724.xml & 1.00 & 1.00 & 1.00 & 1.00 \\
\hline S2504725.xml & 1.00 & 0.24 & 1.00 & 0.24 \\
\hline S2504785.xml & 1.00 & 1.00 & 1.00 & 1.00 \\
\hline S2504811.xml & 0.50 & 1.00 & 0.50 & 1.00 \\
\hline S2504956.xml & 1.00 & 1.00 & 0.82 & 0.82 \\
\hline S2504957.xml & 1.00 & 0.75 & 0.83 & 0.63 \\
\hline S2504988.xml & 0.95 & 0.95 & 0.88 & 0.88 \\
\hline S2504989.xml & 1.00 & 1.00 & 1.00 & 1.00 \\
\hline S2505133.xml & 0.80 & 0.73 & 0.70 & 0.64 \\
\hline S2505483.xml & 1.00 & 1.00 & 0.88 & 0.88 \\
\hline S2505484.xml & 1.00 & 1.00 & 1.00 & 1.00 \\
\hline S2505485.xml & 1.00 & 0.69 & 0.78 & 0.54 \\
\hline S2505505.xml & 1.00 & 1.00 & 1.00 & 1.00 \\
\hline S2505528.xml & 1.00 & 1.00 & 1.00 & 1.00 \\
\hline S2505567.xml & 0.00 & 0.00 & 0.00 & 0.00 \\
\hline $\mathrm{S} 2505569 . \mathrm{xml}$ & 1.00 & 1.00 & 1.00 & 1.00 \\
\hline S2505598.xml & 1.00 & 0.02 & 1.00 & 0.02 \\
\hline S2505663.xml & 1.00 & 1.00 & 0.75 & 0.75 \\
\hline S2505730.xml & 0.00 & 0.00 & 0.00 & 0.00 \\
\hline $\mathrm{s} 2505755 . \mathrm{xml}$ & 0.00 & 0.00 & 0.00 & 0.00 \\
\hline S2506462.xml & 1.00 & 1.00 & 1.00 & 1.00 \\
\hline S2506526.xml & 1.00 & 1.00 & 1.00 & 1.00 \\
\hline s2506561.xml & 1.00 & 0.95 & 1.00 & 0.95 \\
\hline s2506573.xml & 1.00 & 1.00 & 1.00 & 1.00 \\
\hline s2506595.xml & 1.00 & 1.00 & 1.00 & 1.00 \\
\hline s2506659.xml & 1.00 & 0.33 & 1.00 & 0.33 \\
\hline s2506677.xml & 1.00 & 0.60 & 1.00 & 0.60 \\
\hline s2506725.xml & 1.00 & 1.00 & 1.00 & 1.00 \\
\hline S2506777.xml & 1.00 & 0.17 & 1.00 & 0.17 \\
\hline s2506779.xml & 1.00 & 0.33 & 1.00 & 0.33 \\
\hline S2506916.xml & 1.00 & 1.00 & 1.00 & 1.00 \\
\hline S2506958.xml & 1.00 & 1.00 & 1.00 & 1.00 \\
\hline S2507053.xml & 1.00 & 0.85 & 0.93 & 0.79 \\
\hline s2507078.xml & 1.00 & 1.00 & 1.00 & 1.00 \\
\hline s2507194.xml & 1.00 & 1.00 & 1.00 & 1.00 \\
\hline s2507224.xml & 1.00 & 0.67 & 1.00 & 0.67 \\
\hline s2507259.xml & 1.00 & 0.95 & 1.00 & 0.95 \\
\hline s2507280.xml & 1.00 & 0.97 & 0.99 & 0.96 \\
\hline s2507368.xml & 1.00 & 0.75 & 0.67 & 0.50 \\
\hline S2507381.xml & 1.00 & 0.86 & 1.00 & 0.86 \\
\hline s2507397.xml & 1.00 & 0.92 & 1.00 & 0.92 \\
\hline S2507421.xml & 0.00 & 0.00 & 0.00 & 0.00 \\
\hline S2507424.xml & 1.00 & 1.00 & 1.00 & 1.00 \\
\hline s2507522.xml & 1.00 & 0.83 & 0.60 & 0.50 \\
\hline s2507556.xml & 1.00 & 0.65 & 1.00 & 0.65 \\
\hline $\mathrm{s} 2507636 . \mathrm{xml}$ & 1.00 & 1.00 & 1.00 & 1.00 \\
\hline S2507646.xml & 1.00 & 1.00 & 1.00 & 1.00 \\
\hline S2507700.xml & 1.00 & 1.00 & 1.00 & 1.00 \\
\hline S2507732.xml & 1.00 & 1.00 & 1.00 & 1.00 \\
\hline S2507807.xml & 1.00 & 0.50 & 1.00 & 0.50 \\
\hline S2507829.xml & 0.96 & 0.98 & 0.91 & 0.93 \\
\hline S2507833.xml & 1.00 & 0.50 & 1.00 & 0.50 \\
\hline S2507850.xml & 1.00 & 1.00 & 1.00 & 1.00 \\
\hline S2507864.xml & 0.00 & 0.00 & 0.00 & 0.00 \\
\hline S2507868.xml & 1.00 & 0.50 & 0.00 & 0.00 \\
\hline
\end{tabular}




\begin{tabular}{|c|c|c|c|c|}
\hline S2507869.xml & 1.00 & 1.00 & 1.00 & 1.00 \\
\hline S2507870.xml & 0.97 & 0.94 & 0.77 & 0.74 \\
\hline $\mathrm{S} 2507927 . \mathrm{xml}$ & 0.97 & 0.86 & 0.95 & 0.85 \\
\hline S2507945.xml & 1.00 & 1.00 & 1.00 & 1.00 \\
\hline S2507956.xml & 1.00 & 1.00 & 1.00 & 1.00 \\
\hline s2600146.xml & 1.00 & 1.00 & 1.00 & 1.00 \\
\hline s2600982.xml & 0.00 & 0.00 & 0.00 & 0.00 \\
\hline s2601352.xml & 1.00 & 1.00 & 1.00 & 1.00 \\
\hline S2601869.xml & 0.00 & 0.00 & 0.00 & 0.00 \\
\hline S2602251.xml & 1.00 & 1.00 & 1.00 & 1.00 \\
\hline S2602346.xml & 0.00 & 0.00 & 0.00 & 0.00 \\
\hline S2602357.xml & 0.00 & 0.00 & 0.00 & 0.00 \\
\hline S2602372.xml & 1.00 & 1.00 & 1.00 & 1.00 \\
\hline S2602414.xml & 1.00 & 1.00 & 1.00 & 1.00 \\
\hline S2602457.xml & 0.00 & 0.00 & 0.00 & 0.00 \\
\hline S2602657.xml & 1.00 & 1.00 & 1.00 & 1.00 \\
\hline S2602678.xml & 1.00 & 0.67 & 1.00 & 0.67 \\
\hline S2602679.xml & 1.00 & 1.00 & 1.00 & 1.00 \\
\hline S2602716.xml & 1.00 & 1.00 & 1.00 & 1.00 \\
\hline S2602717.xml & 0.00 & 0.00 & 0.00 & 0.00 \\
\hline S2603072.xml & 1.00 & 0.25 & 1.00 & 0.25 \\
\hline S2603179.xml & 1.00 & 1.00 & 1.00 & 1.00 \\
\hline S2603236.xml & 1.00 & 0.70 & 0.86 & 0.60 \\
\hline S2603265.xml & 1.00 & 0.94 & 0.90 & 0.84 \\
\hline S2603362.xml & 1.00 & 0.50 & 1.00 & 0.50 \\
\hline S2603433.xml & 1.00 & 1.00 & 1.00 & 1.00 \\
\hline S2603483.xml & 1.00 & 0.50 & 1.00 & 0.50 \\
\hline S2603484.xml & 1.00 & 1.00 & 1.00 & 1.00 \\
\hline S2603485.xml & 1.00 & 1.00 & 1.00 & 1.00 \\
\hline s2603503.xml & 1.00 & 1.00 & 1.00 & 1.00 \\
\hline S2603576.xml & 1.00 & 1.00 & 1.00 & 1.00 \\
\hline S2603631.xml & 1.00 & 1.00 & 1.00 & 1.00 \\
\hline $\mathrm{s} 2603855 . \mathrm{xml}$ & 1.00 & 1.00 & 1.00 & 1.00 \\
\hline S2603893.xml & 1.00 & 1.00 & 1.00 & 1.00 \\
\hline s2604004.xml & 0.00 & 0.00 & 0.00 & 0.00 \\
\hline s2604161.xml & 0.95 & 0.90 & 0.91 & 0.87 \\
\hline S2604239.xml & 1.00 & 1.00 & 1.00 & 1.00 \\
\hline S2604270.xml & 0.00 & 0.00 & 0.00 & 0.00 \\
\hline S2604282.xml & 1.00 & 0.88 & 0.71 & 0.63 \\
\hline S2604304.xml & 1.00 & 1.00 & 1.00 & 1.00 \\
\hline S2604306.xml & 1.00 & 1.00 & 0.80 & 0.80 \\
\hline s2604329.xml & 1.00 & 1.00 & 1.00 & 1.00 \\
\hline s2604336.xml & 1.00 & 1.00 & 1.00 & 1.00 \\
\hline S2604512.xml & 1.00 & 1.00 & 1.00 & 1.00 \\
\hline S2604596.xml & 1.00 & 1.00 & 1.00 & 1.00 \\
\hline S2604864.xml & 1.00 & 1.00 & 1.00 & 1.00 \\
\hline S2605121.xml & 1.00 & 0.85 & 0.73 & 0.62 \\
\hline S2605122.xml & 1.00 & 1.00 & 1.00 & 1.00 \\
\hline
\end{tabular}

